\title{
Integrin signaling to the actin cytoskeleton
}

Kris A DeMali*, Krister Wennerberg and Keith Burridge

Integrin engagement stimulates the activity of numerous signaling molecules, including the Rho family of GTPases, tyrosine phosphatases, cAMP-dependent protein kinase and protein kinase $\mathrm{C}$, and stimulates production of Ptdlns $(4,5) P_{2}$. Integrins promote actin assembly via the recruitment of molecules that directly activate the actin polymerization machinery or physically link it to sites of cell adhesion.

\section{Addresses}

Department of Cell and Developmental Biology and Lineberger Comprehensive Cancer Center, University of North Carolina, Chapel Hill, NC 27599, USA

*e-mail: kdemali@med.unc.edu

Current Opinion in Cell Biology 2003, 15:572-582

This review comes from a themed issue on

Cell-to-cell contact and extracellular matrix

Edited by Eric Brown and Elisabetta Dejana

0955-0674/\$ - see front matter

(c) 2003 Elsevier Ltd. All rights reserved.

DOI 10.1016/S0955-0674(03)00109-1

$\begin{array}{ll}\text { Abbreviations } & \\ \text { Arp2/3 } & \text { actin-related protein 2/3 } \\ \text { cAMP } & \text { cyclic AMP } \\ \text { CHO } & \text { Chinese hamster ovary } \\ \text { Crk } & \text { chicken tumor virus 10 regulator of kinase } \\ \text { DOCK180 } & \text { 180-kDa protein downstream of CRK } \\ \text { ECM } & \text { extracellular matrix } \\ \text { FAK } & \text { focal adhesion kinase } \\ \text { GEF } & \text { guanine nucleotide exchange factor } \\ \text { GAP } & \text { GTPase-activating protein } \\ \text { GDI } & \text { guanine nucleotide dissociation inhibitor } \\ \text { ILK } & \text { integrin-linked kinase } \\ \text { MAP } & \text { mitogen-activated protein } \\ \text { PAK } & \text { p21-activated protein kinase } \\ \text { PtdIns(4,5)P } & \text { phosphatidylinositol-4,5-bisphosphate } \\ \text { PINCH } & \text { particularly interesting new Cys-His protein } \\ \text { PIP 5-kinase } & \text { phosphatidylinositol 4-phosphate, 5-kinase } \\ \text { PIX } & \text { PAK-interacting exchange factor } \\ \text { PKA } & \text { cAMP-dependent protein kinase } \\ \text { PKC } & \text { protein kinase C } \\ \text { PTP } & \text { protein tyrosine phosphatase } \\ \text { SCAR } & \text { suppressor of cAMP receptor } \\ \text { SHP-2 } & \text { Src homology region 2 containing PTP-2 } \\ \text { SHPS-1 } & \text { SHP substrate-1 } \\ \text { SFK } & \text { Src family kinase } \\ \text { WAVE } & \text { WASP family verprolin-homologous protein } \\ \text { WASP } & \text { Wiskott-Aldrich syndrome protein } \\ & \end{array}$

\section{Introduction}

Twelve years ago, the discovery that integrin engagement stimulates tyrosine phosphorylation of several proteins ushered in an era of extensive research on the signaling that occurs downstream from integrins. Many signaling pathways that emanate from integrin engagement or clustering have been identified and the pace of discovery in this field has not slowed down. This is not surprising given that adhesion to the extracellular matrix (ECM) influences the growth, differentiation, survival, morphology and migratory properties of cells. We suspect that more pathways remain to be uncovered. In this brief review we will restrict our focus to a few selected topics, particularly concentrating on the signaling pathways downstream from integrin engagement that impact on the organization of the cytoskeleton and on cell migration.

\section{Three dimensions versus two}

Although there is a long history of studying the behavior of cells in collagen gels, most work on integrins has involved cells grown on 2D surfaces coated with ECM components derived from serum; these components may be synthesized by the cells themselves or applied by the experimenter. In such cultures, integrins are prominently concentrated in matrix adhesions, which include focal complexes, focal adhesions and fibrillar adhesions. These structures have been discussed elsewhere [1]. Briefly, focal complexes are small transient adhesions at the cell periphery, regulated by Rac or Cdc42. Under the influence of RhoA activity and tension, focal complexes grow in size to become focal adhesions - larger, more stable structures. Fibrillar adhesions - adhesions made to fibronectin fibrils - contain the $\alpha 5 \beta 1$ integrin and a subset of the proteins found in focal adhesions [2]. Of these structures, focal adhesions are often the most pronounced in 2D cultures but are rarely seen in vivo and are much less apparent in cells growing in 3D ECMs [3]. Focal adhesions continue to provide a valuable model for studying the organization of and signaling from relatively stable integrin aggregates, but attention has recently been directed to studying integrin organization and signaling in 3D situations. Unlike cells on 2D surfaces, which have a spread morphology, fibroblasts in 3D matrices develop elongated or stellate morphologies and migrate more rapidly. These cells develop 3D-matrix adhesions that resemble fibrillar adhesions, both in their dimensions and in that the integrin $\alpha 5 \beta 1$ is present, but unlike fibrillar adhesions these matrix adhesions are rich in paxillin, focal adhesion kinase (FAK) and phosphotyrosine [3]. Surprisingly, phosphorylation of the major FAK phosphorylation site (Y397) was not detected, suggesting that the signaling pathways downstream from integrins may differ in 2D and 3D cultures [3].

The physical state of the matrix affects the structure of the adhesions and the morphology of cells, and it is 
possible that this is a major factor contributing to the differences between 2D and 3D cultures. On rigid 2D surfaces focal adhesions are favored [4,5], whereas fibrillar adhesions develop when a pliable fibronectin matrix is remodeled to form fibrils [5]. In 3D collagen gels, fibroblasts at low density display a stellate or dendritic morphology and adhesions to the matrix appear to be diffusely distributed over the cell surface [ $\left.6^{\bullet}\right]$. At high cell density fibroblasts contract the collagen gels, thereby increasing their rigidity. Under these conditions, structures similar or equivalent to focal adhesions develop in a RhoA-dependent manner $\left[6^{\circ}\right]$. These results raise the possibility that the development of a rigid matrix resulting from the initial contractile activity of the cells leads to subsequent isometric tension that may somehow elevate RhoA activity.

\section{Integrin-mediated regulation of Rho family GTPases}

With respect to cytoskeletal organization and cell migration, signaling from integrin-mediated adhesion is typically characterized by two phases. Early adhesion is associated with pathways that stimulate protrusion whereas mature adhesions are associated with the devel- opment of tension. The early phase leads to Rac and Cdc42 activation and to actin polymerization. The later phase leads to RhoA activation, increased contractility and the transmission of tension to the sites of integrin ligation. These pathways are often antagonistic and the biphasic nature and timing of this response can be a source of complexity and confusion.

\section{Regulation of Rac and Cdc42}

During adhesion and spreading on an ECM, cells extend filopodia and lamellipodia, structures regulated by Cdc 42 and Rac, respectively. Integrin-mediated adhesion activates $\mathrm{Cdc} 42$ and $\mathrm{Rac}[7]$ and for Rac this requires an intact $\beta$ integrin subunit $[8,9]$. Rho family GTPases are active when GTP-bound and inactive when bound to GDP. Activation is catalyzed by guanine nucleotide exchange factors (GEFs) and inactivation is promoted by GTPaseactivating proteins (GAPs) that stimulate the intrinsic GTPase activity of the Rho proteins. One example of a GEF activated downstream from integrin engagement is Vav1 [10], but its expression is restricted to hematopoietic cell types. However, the closely related GEF Vav2 is widely distributed and an obvious candidate for activation downstream from integrins (Figure 1). Using tyrosine

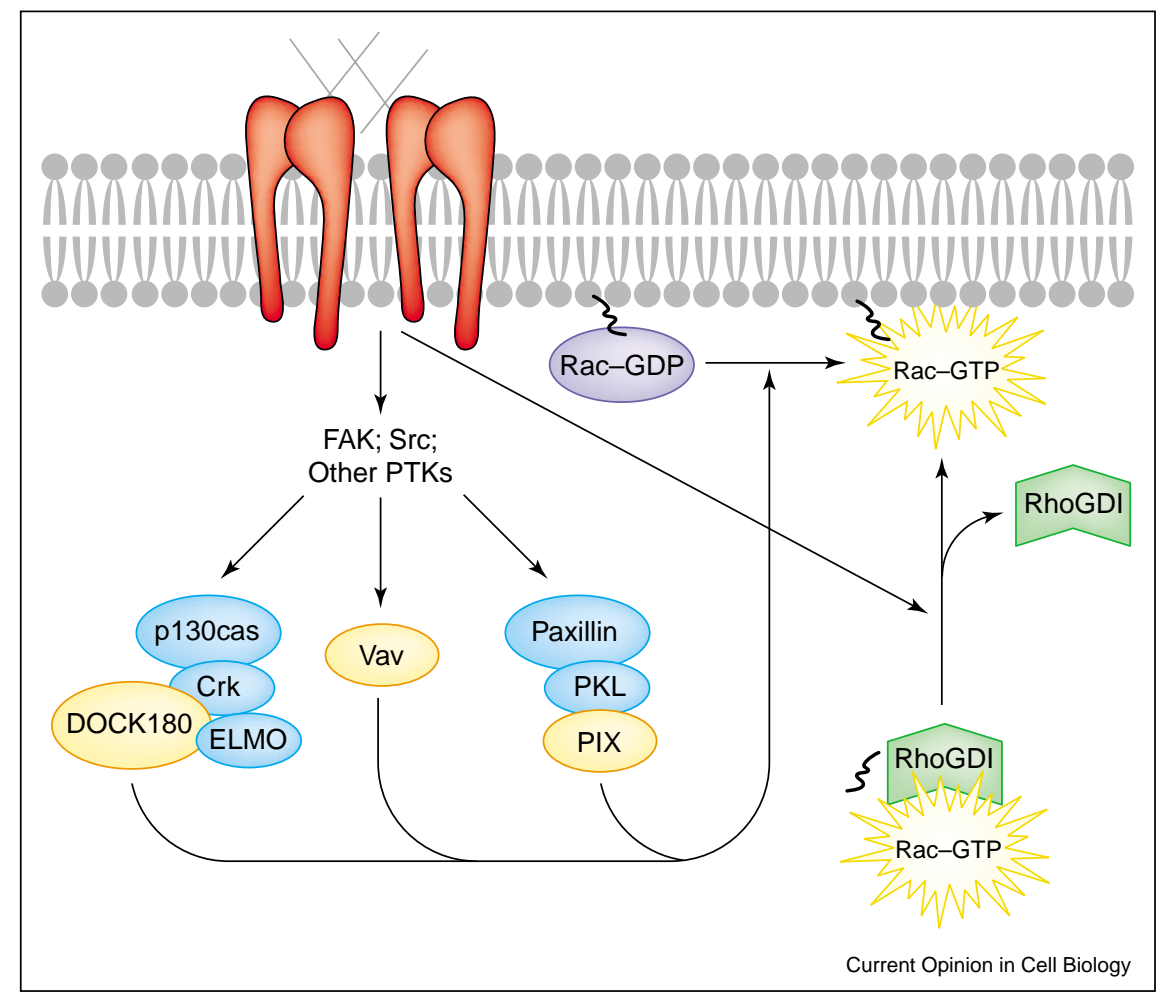

Integrin-mediated activation of Rac and Cdc42. In response to integrin engagement, several tyrosine kinases are activated, including FAK and Src. These tyrosine kinases phosphorylate substrates, leading to the activation of Rac and Cdc42 (only Rac is indicated). The phosphorylated proteins include the following: GEFs (yellow circles), which activate Rac and Cdc42, such as Vav; adaptor protein complexes (blue circles) such as paxillin and PKL or p130Cas, Crk, and ELMO that bind GEFs such as PIX or DOCK180. Alternatively, integrins trigger translocation of RhoGDI-bound Rac-GTP to the plasma membrane where active Rac is liberated and available to interact with effectors. 
phosphorylation as an indicator of activation, evidence was presented that Vav2 is activated downstream from growth factor receptors but not from integrins [11,12]. Nevertheless, a dominant-negative form of Vav2 blocked lamellipodium formation and spreading on fibronectin, which is consistent with Vav2 having a role in Rac activation following integrin engagement [13]. Interestingly, an elevation in tyrosine phosphorylation was not seen in response to adhesion, which is consistent with the earlier work and suggests that changes to multiple phosphorylation sites might have masked an elevation in phosphorylation of the site(s) associated with activation, or that Vav2 activation occurs by other means.

Other pathways downstream of integrins that lead to Rac activation may also be involved. Both p130cas and paxillin associate with FAK and both have been linked to Rac activation. Tyrosine phosphorylation of p130cas promotes a complex of Crk, ELMO and DOCK180 [14-18,19 ${ }^{\bullet \bullet}$ ] (Figure 1). Recent work has revealed that DOCK180 is a Rac GEF, even though it lacks the Dbl-homology/pleckstrin-homology tandem domains characteristic of conventional Rho-family GEFs $\left[19^{\bullet \bullet}, 20^{\bullet \bullet}\right]$. Another complex also associates with paxillin: this complex includes PKL (GIT) and Pak-interacting exchange factor (PIX), the latter being a conventional Rac GEF (reviewed in [21]) (Figure 1). Deciphering the relative importance of the different pathways that potentially lead to Rac activation following integrin engagement will be important.

Integrin-mediated activation of Rac and other Rho-family proteins may involve not only GEF activation but also targeting of the GTP-bound protein to sites of adhesion. Del Pozo and coworkers have found that a fraction of active Rac is sequestered by RhoGDI and that this active Rac is selectively released close to sites of integrinmediated adhesion, allowing it to interact with effectors in this region of the cell $\left[22^{\bullet \bullet}\right]$. It will be interesting to determine how this is achieved and whether localized release of active Rho family members from RhoGDI is a general mechanism.

\section{Regulation of RhoA}

Integrin engagement leads to a transient depression in RhoA activity [23] and it has been argued that this promotes lamellipodial extension during cell migration [24]. The dip in RhoA activity requires Src, FAK and p190RhoGAP [25,26]. A role for paxillin phosphorylation has also been indicated in this decrease in RhoA activity. When two of the paxillin phosphorylation sites (Y31 and Y118) were mutated, the depression in RhoA activity was abolished and the cells showed premature formation of stress fibers $\left[27^{\circ}\right]$. These authors demonstrated that the phosphorylation of these two tyrosines, which is induced by integrin-mediated adhesion, generates a binding site for p120RasGAP, displacing it from its binding partner p190RhoGAP. Evidence was presented that p190Rho-
GAP freed from p120RasGAP was activated and hence contributed to the decrease in RhoA activity [27 $\left.{ }^{\bullet}\right]$. However, whether the interaction of p120RasGAP with p190RhoGAP inhibits or increases the latter's activity remains controversial. Interestingly, the decrease in RhoA activity is seen even with cells in suspension that bind soluble-peptide integrin ligands [25], a situation in which FAK does not become activated and paxillin does not become phosphorylated on these tyrosine residues. This suggests that the phosphorylation of paxillin cannot be the sole mechanism of regulation and that phosphorylation of p190RhoGAP may also be important [25]. Nevertheless, paxillin phosphorylation may contribute to the depression of RhoA activity when cells adhere to fibronectin, which results in a more robust inhibition of the RhoA response than is seen in cells in suspension stimulated with soluble ligands.

Examination of the time-course of RhoA activity in response to cells adhering to fibronectin reveals that the initial dip is followed by activation [23]. Engagement of non-integrin receptors such as syndecan-4 may contribute to this response [28-30], but integrins have also been observed to contribute to activation. Here, different responses have been observed with different integrins. O'Connor and colleagues observed that engagement or clustering of $\alpha 6 \beta 4$ resulted in stimulation of RhoA activity, in contrast to the depression induced by clustering $\beta 1$ integrins [31]. Engagement of $\alpha \mathrm{v} \beta 3$ on astrocytes by Thy-1 was shown to stimulate assembly of focal adhesions and stress fibers, which is consistent with RhoA activation occurring downstream from this integrin [32]. Direct evaluation of the effect of $\beta 1$ and $\beta 3$ integrins on RhoA activity was performed in Chinese hamster ovary $(\mathrm{CHO})$ cells in which these integrins were overexpressed $\left[33^{\circ}\right]$. In this system, overexpression of $\beta 3$ resulted in a pronounced increase in Rho-GTP levels when the cells were plated on fibronectin or fibrinogen, whereas $\beta 1$ overexpression had no effect. Somewhat surprisingly, expression of a $\beta 1 / \beta 3$ chimera in which a heptapeptide sequence from the $\beta 1$ extracellular-I-domain-like structure was replaced by the equivalent sequence from the $\beta 3$ integrin resulted in stimulation of Rho activity [33 $3^{\circ}$. A different result was found using cells deficient in $\beta 1$ integrins $\left[3^{\bullet \bullet}\right]$. Using either GD25 or GE11 cells, reexpression of $\beta 1$ subunits stimulated RhoA activity, whereas $\beta 3$ had no effect. Although the results were the opposite in the two studies, both studies found that the extracellular domain was critical. A possible explanation for the opposite results is the different cell types used by these two groups. It is easy to imagine that the requirements of a particular integrin may differ in different cell types, and that in some situations, but not others, it is advantageous for the integrin to be coupled to Rho activation. The large number of RhoGEFs and their variable expression in different cell types may provide cell-type specificity when coupling integrins to Rho 
activation. The significance of the extracellular domains of the integrins in this coupling remains unclear.

\section{Integrins and protein tyrosine phosphatases}

Integrin-mediated adhesion induces the tyrosine phosphorylation of many proteins. The consequences of this tyrosine phosphorylation and the kinases involved have commanded much attention. By comparison, the protein tyrosine phosphatases (PTPs) have been much less studied, although several recent papers suggest that this is changing. Early work indicated that integrin-mediated adhesion results in a bulk inhibition of PTP activity that parallels the increase in tyrosine phosphorylation seen in response to adhesion [35]. Sastry and coworkers, however, have found that PTP-PEST is stimulated upon integrinmediated adhesion [ $\left.36^{\circ}\right]$. This PTP had previously been shown to act on various focal adhesion targets such as p130cas [37] and paxillin [38], but not on FAK. Both overexpression and deletion of PTP-PEST inhibit cell migration $[39,40]$, suggesting that a fine balance in the level of tyrosine phosphorylation of relevant substrates regulates cell migration. PTP-PEST overexpression inhibits protrusive activity and this has been related to a depression in Rac activity [36 $6^{\circ}$. As mentioned above, the tyrosine phosphorylation of both p130cas and paxillin has been linked to Rac activation, and so PTP-PEST may be affecting Rac activity by dephosphorylating these known targets.

One PTP that has been associated both with integrinmediated signaling and with regulating RhoA activity is SHP-2 (Src homology region 2 containing PTP-2). Perturbation of SHP-2 levels or activity has effects on adhesion, cytoskeletal organization and cell migration [41-45].
Conflicting results have been obtained with respect to SHP-2's effect on Rho activity, with some groups detecting activation $[45,46]$ and others inhibition $[47,48]$. It is difficult to reconcile these differences; however, it is possible that in different cellular contexts SHP-2 acts on different targets that influence RhoA activity in opposite directions (Figure 2). Recent work has identified p190BRhoGAP as a potential target for SHP-2 [49*0]. Here tyrosine phosphorylation has been associated with increased GAP activity and so the action of SHP-2 to decrease p190 activity will result in elevated RhoA activity $\left[49^{\bullet \bullet}\right.$. By contrast, the activity of some GEFs (e.g. the Vav family, PDZ-RhoGEF and leukemia-associated RhoGEF) is stimulated by tyrosine phosphorylation [50-52]. Although Vav2 has broad specificity for Rho family GTPases in vitro, in vivo the phenotype resulting from activated Vav2 varies with cell type, often suggesting increased Rac activity but at other times increased RhoA activity as well [12]. SHP-2 will exert an inhibitory effect on RhoGEFs that are stimulated by tyrosine phosphorylation.

One of the substrates for SHP-2 is the transmembrane protein SHP substrate 1 (SHPS-1, also known as SIRP $\alpha 1$ ), which becomes tyrosine-phosphorylated in response to integrin-mediated adhesion by FAK and Src family kinases [53]. SHPS-1 binds SHP-2, thereby targeting SHP-2 to the membrane, where it may act on other tyrosine-phosphorylated proteins. Expression of a truncated form of SHPS-1 lacking most of the cytoplasmic domain and unable to bind SHP-2 results in cells with increased stress fibers [45]. Contrary to expectations these cells exhibit reduced rather than elevated RhoA activity [45]. The reason for this paradoxical result has not been resolved.

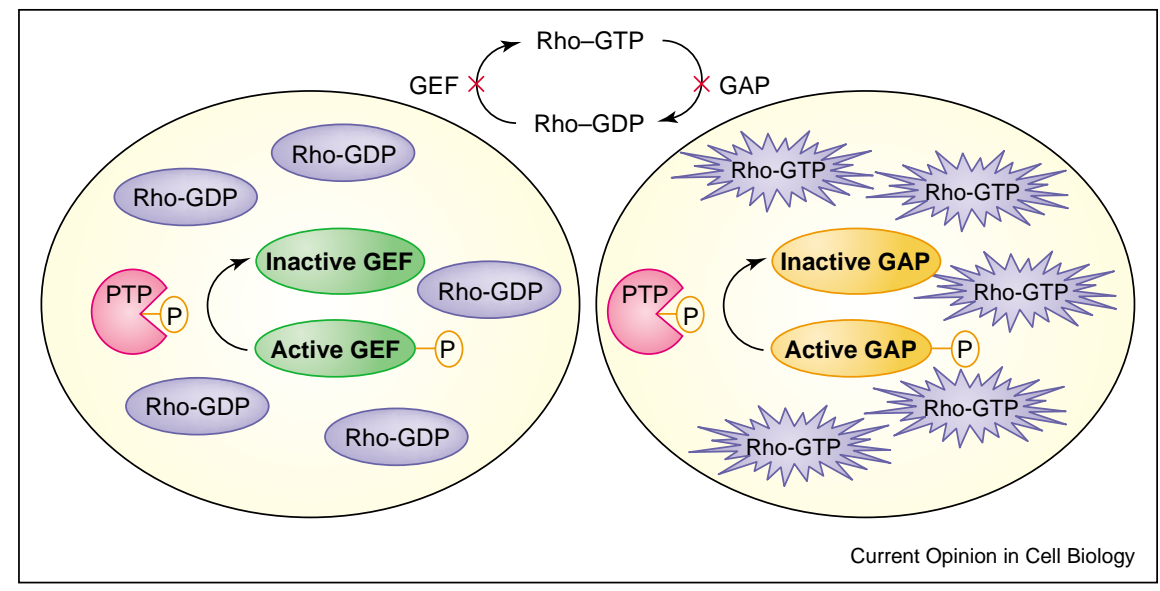

Model for the potential regulation of Rho activity by tyrosine phosphatases. Left: a tyrosine phosphorylated activated GEF (green ovals) is dephosphorylated by a tyrosine phosphatase (shown in pink). The GEF is unable to catalyze the exchange of GTP for GDP and Rho is left in the inactive GDP-bound form (purple circles). Right: the phosphatase dephosphorylates a GAP (orange ovals), inactivating it, and Rho-GTP levels accumulate (purple starbursts). 
In some situations, engagement of integrins with their ligands promotes integrin association with lipid rafts (reviewed in [54]). SHP-2 is targeted to rafts in response to integrin binding to its ligands [55 $5^{\bullet \bullet}$. Significantly, the use of a double palmitoylation signal to target SHP-2 to lipid rafts in cells in suspension stimulates FAK tyrosine phosphorylation and other pathways normally triggered by integrin engagement $\left[5^{\circ \bullet}\right]$. Targeting SHP-2 to lipid rafts also affected Rho activity: the resting level of active Rho was elevated, but a dip in activity was still observed in response to adhesion to fibronectin. Interestingly, when a catalytically dead form of SHP-2 was targeted to lipid rafts, the level of Rho activity returned to more normal levels, but the adhesion-induced dip was abolished. These results led the authors to conclude that SHP-2 can function as both a positive and negative regulator of RhoA activity [55*0. The elevation of Rho activity by SHP-2 in lipid rafts could be explained by the regulation of p190RhoGAP phosphorylation and activity $\left[49^{\circ \bullet}\right.$. However, the fact that catalytically inactive SHP-2 blocks the adhesion-induced dip in Rho activity suggests that p190RhoGAP is not becoming activated in this situation. It would be possible to explain this observation if, in response to integrin engagement, SHP-2 promotes Src activation by removing Src's inhibitory C-terminal phosphate [43]. The catalytically inactive SHP-2 would block this pathway and thereby prevent the tyrosine phosphorylation of $\mathrm{p}$ 190RhoGAP and consequent depression of Rho activity. The different observations obtained with SHP-2 illustrate the complexity of these pathways and how P'TPs may act at multiple sites in a pathway, often generating paradoxical results (illustrated in Figure 2).

PTP $\alpha$, another PTP implicated in promoting cell spreading [56] and found in focal adhesions [57], removes inhibitory phosphates from the C-terminal tyrosines of Src family kinases (SFKs) [56,58]. Cells lacking PTP $\alpha$ reveal decreased FAK phosphorylation, particularly on tyrosine 397 , the autophosphorylation site, leading to the suggestion that $\mathrm{PTP} \alpha$ activity lies between integrin engagement and FAK activation in the pathway [59 $\left.{ }^{\bullet}\right]$. Strong support for this idea comes from another study in which $\mathrm{PTP} \alpha$ has been found to physically associate with the integrin $\alpha v \beta 3$ but not with $\alpha 5 \beta 1\left[60^{\bullet \bullet}\right]$. This work built on earlier studies showing that $\alpha \mathrm{v} \beta 3$ integrin signaling via a Src family kinase is involved in the reinforcement of integrin-cytoskeleton linkages [61]. The decrease in focal adhesions seen in the PTP $\alpha$ null cells, together with their decreased development of force transmitted to $\alpha v \beta 3$ integrins $\left[60^{\bullet \bullet}\right.$, suggests that $\mathrm{PTP} \alpha$ may normally contribute to the activation of RhoA downstream from $\alpha v \beta 3$ integrin engagement. In preliminary work, our laboratory has confirmed that $\mathrm{PTP}^{\mathrm{T}} \alpha$ null cells exhibit decreased RhoA-GTP levels when plated on fibronectin (Ellerbroek and Burridge, unpublished observations). As mentioned earlier, in some cells $\beta 3$ integrin engagement

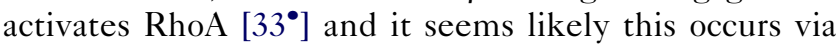

a P'TP $\alpha /$ SF K pathway. At first, this seems to conflict with the idea that SFKs downstream from integrin engagement depress RhoA activity via p190RhoGAP [25]; however, an explanation is suggested by the finding of von Wichert and coworkers that it is Fyn rather than Src that becomes activated by PTP $\alpha$ downstream of $\alpha \mathrm{v} \beta 3$ integrin occupancy $\left[60^{\bullet \bullet}\right]$. These investigators found that overexpression of $\mathrm{Src}$ in cells expressing $\mathrm{PTP} \alpha$ actually depressed focal-adhesion formation, whereas this was not seen with Fyn $\left[60^{\bullet \bullet}\right]$. Together these results suggest a model in which the initial depression of RhoA activity occurs via integrin-mediated activation of Src leading to elevated p190RhoGAP activity, whereas the slower increase in RhoA activity occurs as a result of PTP $\alpha$ 's activation of Fyn (Figure 3). Presumably this involves a RhoGEF that is stimulated by tyrosine phosphorylation. It will be interesting to determine whether other integrins may also couple to PTP $\alpha$ in some situations or cell types, and whether this may account for the elevation of RhoA

\section{Figure 3}

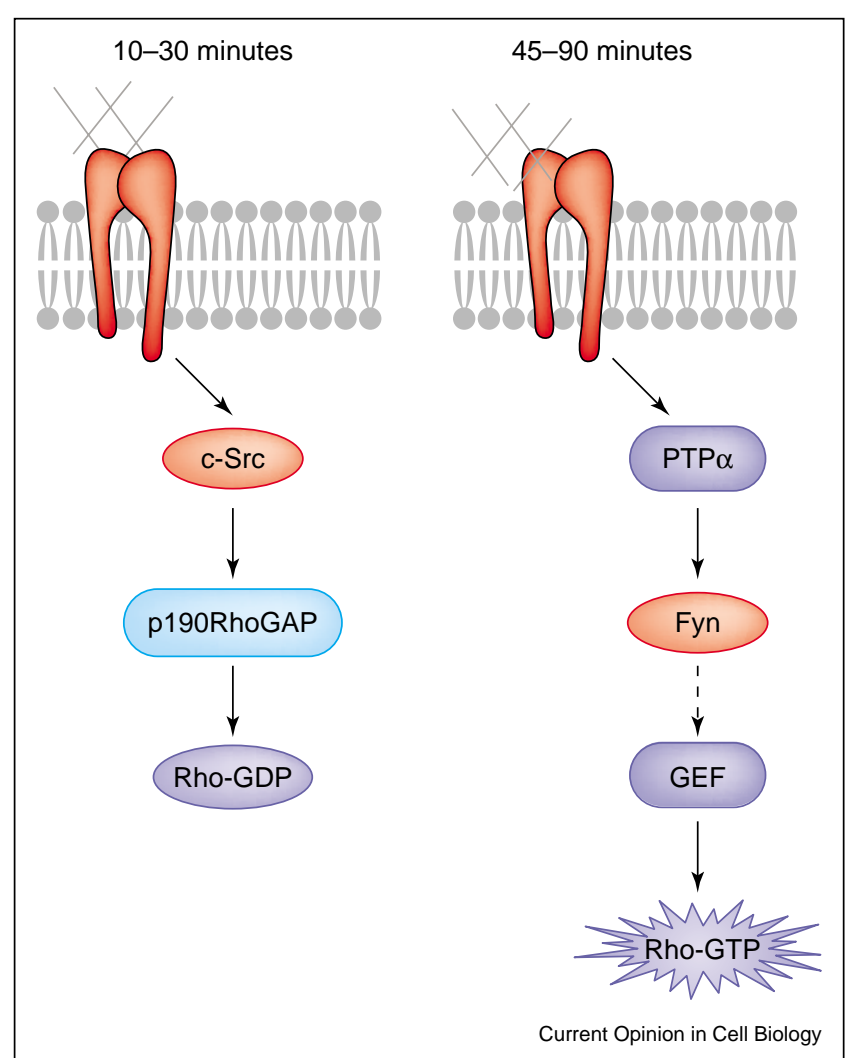

Role of Src family kinases in the inhibition and reactivation of Rho activity. During the first 10-30 minutes of adhesion via some integrins, the activity of Rho is transiently suppressed. The pathway to inhibition involves c-Src-dependent phosphorylation and activation of p190RhoGAP. This GAP triggers the hydrolysis of GTP bound to Rho rendering it inactive. With other integrins, or with the same integrins at later times (45-90 minutes) of adhesion, the levels of Rho-GTP increase as a result of PTP $\alpha$ activating the tyrosine kinase, Fyn, which presumably phosphorylates and activates a RhoGEF. 
activity downstream from $\alpha 5 \beta 1$ integrins observed by Danen and coworkers $\left[34^{\bullet \bullet}\right]$.

\section{Integrin regulation of CAMP/PKA}

The activity of cAMP-dependent protein kinase (PKA) regulates the cytoskeleton both by inhibiting contractility and by stimulating protrusion. PKA can inhibit actinmyosin contractility in several ways. It can phosphorylate the $G \alpha_{13}$ subunit of heterotrimeric $G$ proteins, leading to decreased downstream RhoGEF activation [62]; it can directly phosphorylate the $\mathrm{C}$ terminus of RhoA, resulting in an increased binding to RhoGDI and thereby terminate RhoA activity [63,64]; and finally, it can phosphorylate and inactivate myosin light chain kinase [65]. All these behaviors lead to decreased actin-myosin contractility. In addition, PKA has been shown to phosphorylate and inhibit vasodilator-stimulated phosphoprotein (VASP) [66] and p21-activated protein kinase (PAK) [67], and to activate Rac1 and Cdc42 [68,69]. Increased levels of cAMP or activation of PKA in cells inhibit RhoA activation [70] and lead to loss of stress fibers and focal adhesions [63]. Conversely, inhibition of PKA results in formation of stress fibers [71,72] and allows adhesion-independent mitogenactivated protein (MAP) kinase activation [66].

While PKA regulates adhesion, integrin ligation and cell adhesion in turn are potent regulators of PKA activity. Detachment of cells causes a transient activation of PKA [66], probably through a relaxation-dependent mechanism [73]. The detachment-dependent activation of PKA prevents anchorage-independent activation of MAP kinase by inactivating PAK [67]. PKA activity in suspended cells, however, returns to baseline levels within 60-90 minutes. Somewhat surprisingly, adhesion will also activate PKA $[66,68]$. The time-course of this correlates with the activation of Rac and Cdc42 [7,74], the inactivation of RhoA [23], and the timing of membrane protrusions during cell spreading. Similarly, ligand-dependent clustering of $\beta 1$ integrins by function-blocking $\beta 1$ antibodies or soluble-peptide integrin ligands induces activation of PKA $[75,76]$. As with the detachment-dependent activation of PKA, the attachment-dependent activation of PKA is transient $[66,68]$ and the later reduction in PKA activity allows cells to form stress fibers and focal adhesions, adhere firmly, and sustain survival signals, possibly through MAP kinase signaling [67,71,72]. The deactivation, at least in endothelial cells, is matrix- and integrindependent: integrins $\alpha 1 \beta 1, \alpha 2 \beta 1$, or $\alpha 5 \beta 1$ can support inactivation but $\alpha 6 \beta 1$ or $\alpha \mathrm{V} \beta 3$ cannot $[71,72]$.

\section{An interplay between integrins and protein kinase C}

Protein kinase $\mathrm{C}(\mathrm{PKC}) \alpha$ was one of the first signaling molecules identified in focal adhesions [77] and subsequent work has established that isoforms of PKC become activated following adhesion to the ECM and cell spreading [78-80,81 ${ }^{\bullet}$. For example, in muscle cells activation of
PKC $\varepsilon$ is followed sequentially by activation of PKC $\alpha$ and $\operatorname{PKC} \delta\left[81^{\circ}\right]$. Whereas activation of PKC promotes cell spreading and focal adhesion formation in fibroblasts [28], it should be noted that PKC stimulation (e.g. by phorbol esters) induces disruption of focal adhesions in epithelial cells $[82,83]$. In fibroblasts, early work established that adhesion to the cell-binding domain of fibronectin mediated by $\alpha 5 \beta 1$ is insufficient for cells to develop focal adhesions, but assembly of these structures could be stimulated by PKC activation or by the addition of the heparin-binding domain of fibronectin (reviewed in [84]). The relevant proteoglycan responsible for promoting focal adhesion assembly was identified as syndecan-4, a transmembrane proteoglycan that binds to the heparin-binding domain of fibronectin (reviewed in [84]). Syndecan-4 localizes to focal adhesions and PKC $\alpha$ binds to syndecan-4's cytoplasmic domain (reviewed in [84]). Recent work has revealed an interesting level of complexity. Whereas the integrin $\alpha 5 \beta 1$ requires PKC $\alpha$ activation via syndecan- 4 for focal adhesions to develop, the integrin $\alpha 4 \beta 1$, which binds to another site in fibronectin, does not $\left[85^{\bullet \bullet}\right]$. Other interesting differences exist between these two integrins, such as the binding of paxillin by the $\alpha 4$ cytoplasmic domain [86], which prompts the question of whether the recruitment of paxillin or some other protein by $\alpha 4 \beta 1$ fulfills functions that may be supplied by PKC $\alpha$ activation downstream from $\alpha 5 \beta 1$ engagement. Downstream from syndecan-4 engagement, multiple studies have implicated RhoA activation as well as PKC activation $[29,87]$, raising the possibility that PKC may be upstream of RhoA. This has recently been validated with the demonstration that the RhoA-GEF p115 is a substrate for and stimulated by PKC $\alpha$ [88].

\section{Regulation of Ptdlns $(4,5) P_{2}$ by integrin signaling}

The activities of many cytoskeletal proteins are regulated by phosphatidylinositol-4,5-bisphosphate $\left(\operatorname{Ptd} \operatorname{Ins}(4,5) P_{2}\right)$. Downstream from integrins, both vinculin and talin undergo a conformational change on binding this molecule. With vinculin, this exposes cryptic binding sites for other proteins, including talin [89-91], whereas talin's interaction with $\operatorname{PtdIns}(4,5) P_{2}$ promotes its binding to the cytoplasmic domain of $\beta 1$ integrin subunit [92]. Significantly, integrin-mediated adhesion stimulates PtdIns $(4,5) P_{2}$ synthesis [93]. In part, this may occur via stimulation of PI5-kinase by Rho and Rac, although the mechanism by which this could occur is not well understood. Recent work has revealed another pathway. Independently, two groups have found that one PIP 5-kinase splice isoform localizes to focal adhesions by binding to talin and that this interaction stimulates its activity $\left[94^{\bullet \bullet}, 95^{\bullet \bullet}\right]$. In one case, the direct interaction was found to stimulate PIP 5-kinase activity, whereas in the other case localization to focal adhesions resulted in activation by FAK phosphorylation. This phosphorylation of PIP 
5-kinase not only stimulated catalytic activity but also

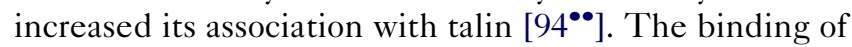
PIP 5-kinase to talin and its consequent activation should elevate PtdIns $(4,5) P_{2}$ levels in the local region where integrins are clustered. The positive feedback mechanism suggested by these findings should contribute to the assembly of these integrin-based complexes. Indeed, expression of a kinase-dead form of the relevant PIP 5-kinase isoform blocked recruitment of FAK to focal adhesions. The local increase in $\operatorname{PtdIns}(4,5) P_{2}$ in the vicinity of integrin engagement may also stimulate actin polymerization, as discussed below.

\section{Regulation of actin assembly by integrins}

Several of the prominent integrin-associated structural proteins (talin, vinculin and $\alpha$-actinin) bind $\mathrm{F}$-actin, but relatively little polymerization of actin has been detected in focal adhesions. As mentioned above, localized PtdIns $(4,5) P_{2}$ synthesis in focal adhesions may contribute to the assembly of protein complexes and the binding of actin at these sites. It may also promote the limited polymerization that occurs in focal adhesions by dissociating capping proteins from the barbed ends of actin filaments [96] or by other mechanisms. Much more polymerization of actin occurs at the leading edge of cells, which is also the region where integrins first engage their ligands. The major nucleator of actin polymerization is the actin-related protein $2 / 3$ (Arp2/3) complex. Recent work has established a link between the Arp $2 / 3$ complex and new sites of integrin engagement that is mediated by the Arp $2 / 3$ complex binding to vinculin [97 ${ }^{\bullet}$ (Figure $4 a$ ). This interaction is transient, being confined to the newest adhesions and not seen in more mature focal adhesions.
The association is regulated by phosphatidylinositol-3kinase and Rac activity. Cells deficient in vinculin show decreased spreading and formation of lamellipodia, phenotypes corrected by re-expression of wildtype vinculin but not of vinculin unable to bind the Arp $2 / 3$ complex $\left[97^{\circ}\right]$. Although this interaction does not stimulate actin polymerization by the Arp $2 / 3$ complex, it does recruit the Arp2/3 complex to sites of integrin clustering.

Other mechanisms for linking actin polymerization to integrins have also been identified. Key regulators of the Arp2/3 complex are members of the Wiskott-Aldrich syndrome protein (WASP) family of proteins, including WASP, N-WASP and WAVE/SCAR proteins. N-WASP has been identified in $\beta 1$-integrin immunoprecipitates and was released under conditions stimulating actin polymerization and protrusion [98]. The WASP proteins stimulate the Arp2/3 complex after they have undergone a conformational change in which the $\mathrm{C}$-terminal domain is exposed, enabling it to bind the Arp $2 / 3$ complex. WASP and N-WASP are activated by binding to Cdc 42 or Nck, whereas WAVE/SCAR is activated downstream from Rac or Nck $\left[99,100,101^{\bullet \bullet}\right.$. Nck also binds to WIP, a WASPinteracting protein that promotes actin polymerization [102]. A link between Nck and integrins has been identified via the LIM domain protein, PINCH, which binds integrin-linked kinase (ILK), which in turn associates with integrin $\beta$ subunit cytoplasmic domains [103] (Figure 4b). Significantly, the action of WASP on Arp2/ 3 -induced actin polymerization is stimulated by PtdIns $(4,5) P_{2}$, which, as mentioned above, is synthesized by enzymes recruited to sites of integrin engagement by binding talin $\left[94^{\bullet \bullet}, 95^{\bullet \bullet}\right]$. (a)

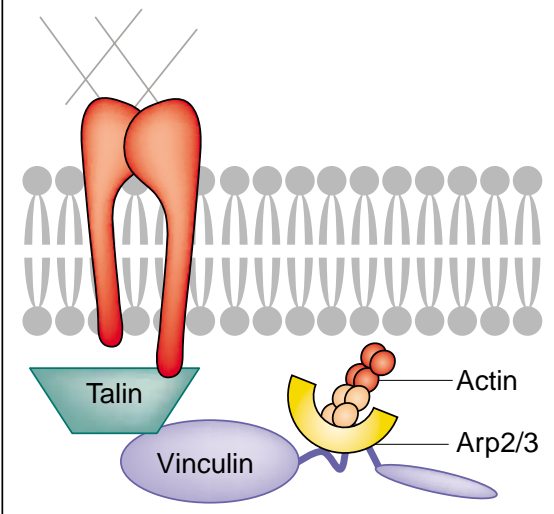

(b)

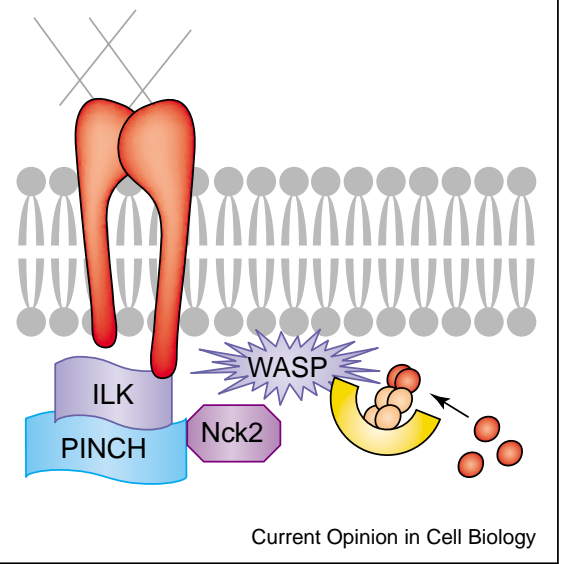

Links between the actin polymerization machinery and integrins. (a) Activated Arp2/3 complex binds directly to the hinge region of vinculin, an adhesion molecule that is recruited to integrins via an interaction with talin. Binding of the Arp $2 / 3$ complex to vinculin does not stimulate the activity of the Arp2/3 complex, but rather localizes polymerization to new sites of integrin adhesion. (b) Actin polymerization is stimulated at sites of integrin clustering via recruitment of a complex of proteins, including ILK-PINCH and Nck. Nck binds and activates WASP proteins, which in turn recruit and activate the Arp2/3 complex. 
The above observations suggest that the machinery for nucleating actin polymerization can be linked in various ways to integrins and may be particularly active where integrins are newly engaged with the ECM. Superimposed on these physical links between integrins and the Arp $2 / 3$ complex is the activation of Rac and Cdc42 downstream from integrin ligation. The local activation of these GTPases will further stimulate WASP or WAVE/ SCAR in the vicinity of integrin-ligand binding. Additionally, selective release of activated Rac from the sequestering protein RhoGDI has been reported to occur where integrins mediate adhesion to the ECM [22*0]. Together, these pathways should synergize to promote actin polymerization at sites of new adhesion.

\section{Conclusions}

Progress in the field of integrin-mediated signaling has been substantial in the last couple of years, but much still remains to be learned. The apparent complexity of many of the signaling pathways downstream from integrin ligation reflects in part the transition from an early response, associated with $\mathrm{Rac} / \mathrm{Cdc} 42$ activation and membrane protrusion, to a late response, associated with RhoA activation and the generation of tension. The development of live cell imaging techniques should contribute to resolving many of the spatial and temporal complexities downstream from integrin engagement. Biosensors are being designed and tested that will visualize specific signaling events, such as activation of Rho GTPases, kinases and phosphatases, within living cells in real time. This technology promises to revolutionize the field by allowing signaling pathways to be visualized locally within cells as integrin ligation occurs, matures and is terminated.

\section{Acknowledgements}

We would like to express our gratitude to Leslie Parise, Mike Schaller, Alan Howe and members of the Burridge Laboratory for critical reading of the manuscript. This work was supported by NIH grants \#GM29860, HL45100, and postdoctoral fellowship GM20610.

\section{References and recommended reading}

Papers of particular interest, published within the annual period of review, have been highlighted as:

- of special interest

$\bullet$ of outstanding interest

1. Geiger B, Bershadsky A, Pankov R, Yamada KM: Transmembrane cross-talk between the extracellular matrix - cytoskeleton cross-talk. Nat Rev Mol Cell Biol 2001, 2:793-805.

2. Zamir E, Geiger B: Molecular complexity and dynamics of cell-matrix adhesions. J Cell Sci 2001, 114:3583-3590.

3. Cukierman E, Pankov R, Stevens DR, Yamada KM: Taking cell-matrix adhesions to the third dimension. Science 2001, 294:1708-1712.

4. Pelham RJ Jr, Wang Y: Cell locomotion and focal adhesions are regulated by substrate flexibility. Proc Natl Acad Sci USA 1997, 94:13661-13665

5. Katz BZ, Zamir E, Bershadsky A, Kam Z, Yamada KM, Geiger B: Physical state of the extracellular matrix regulates the structure and molecular composition of cell-matrix adhesions. Mol Biol Cell 2000, 11:1047-1060.

6. Tamariz E, Grinnell F: Modulation of fibroblast morphology and - adhesion during collagen matrix remodeling. $\mathrm{Mol} \mathrm{Biol} \mathrm{Cell}$ 2002, 13:3915-3929.

This paper investigates how cell adhesion is altered as matrix remodeling progresses in three dimensions. They find that as fibroblasts encounter resistance in a collagen matrix, and their overall morphology changes from dendritic to bipolar, cell-matrix adhesions change from punctate to focal adhesions. Rho kinase activity is needed for these changes to occur.

7. Price LS, Leng J, Schwartz MA, Bokoch GM: Activation of Rac and Cdc42 by integrins mediates cell spreading. Mol Biol Cell 1998, 9:1863-1871.

8. Berrier AL, Martinez R, Bokoch GM, LaFlamme SE: The integrin $\beta$ tail is required and sufficient to regulate adhesion signaling to Rac1. J Cell Sci 2002, 115:4285-4291.

9. Hirsch E, Barberis L, Brancaccio M, Azzolino O, Xu D, Kyriakis JM, Silengo L, Giancotti FG, Tarone G, Fassler R et al.: Defective Racmediated proliferation and survival after targeted mutation of the $\beta 1$ integrin cytodomain. J Cell Biol 2002, 157:481-492.

10. Miranti CK, Leng L, Maschberger P, Brugge JS, Shattil SJ: Identification of a novel integrin signaling pathway involving the kinase Syk and the guanine nucleotide exchange factor Vav1. Curr Biol 1998, 8:1289-1299.

11. Moores SL, Selfors LM, Fredericks J, Breit T, Fujikawa K, Alt FW, Brugge JS, Swat W: Vav family proteins couple to diverse cell surface receptors. Mol Cell Biol 2000, 20:6364-6373.

12. Liu BP, Burridge K: Vav2 activates Rac1, Cdc42, and RhoA downstream from growth factor receptors but not $\beta 1$ integrins Mol Cell Biol 2000, 20:7160-7169.

13. Marignani PA, Carpenter CL: Vav2 is required for cell spreading $J$ Cell Biol 2001, 154:177-186.

14. Cary LA, Han DC, Polte TR, Hanks SK, Guan JL: Identification of p130Cas as a mediator of focal adhesion kinase-promoted cell migration. J Cell Biol 1998, 140:211-221.

15. Klemke RL, Leng J, Molander R, Brooks PC, Vuori K, Cheresh DA: CAS/Crk coupling serves as a "molecular switch" for induction of cell migration. J Cell Biol 1998, 140:961-972.

16. Matsuda M, Ota S, Tanimura R, Nakamura H, Matuoka K, Takenawa T, Nagashima K, Kurata T: Interaction between the amino-terminal SH3 domain of CRK and its natural target proteins. J Biol Chem 1996, 271:14468-14472.

17. Hasegawa H, Kiyokawa E, Tanaka S, Nagashima K, Gotoh N, Shibuya M, Kurata T, Matsuda M: DOCK180, a major CRKbinding protein, alters cell morphology upon translocation to the cell membrane. Mol Cell Biol 1996, 16:1770-1776.

18. Vuori K, Hirai H, Aizawa S, Ruoslahti E: Introduction of p130cas signaling complex formation upon integrin-mediated cell adhesion: a role for Src family kinases. Mol Cell Biol 1996, 16:2606-2613.

19. Brugnera E, Haney L, Grimsley C, Lu M, Walk SF,

•• Tosello-Trampont AC, Macara IG, Madhani H, Fink GR, Ravichandran KS: Unconventional Rac-GEF activity is mediated through the Dock180-ELMO complex. Nat Cell Biol 2002, 4:574-582

This paper, and [20*0], identify Dock180, a protein lacking a conventional $\mathrm{Dbl}$ homology/Pleckstrin homology tandem domain, as an activator of Rac. Evidence is presented that Dock180 binds ELMO and stimulates the GTP-loading of Rac.

20. Cote JF, Vuori K: Identification of an evolutionarily conserved -. superfamily of DOCK180-related proteins with guanine nucleotide exchange activity. J Cell Sci 2002, 115:4901-4913. The authors show that DOCK180 belongs to a family of exchange factors that bind to nucleotide-free Rac and that it catalyzes the exchange of GDP for GTP on Rac. In contrast to Brugnera et al. [19**], they present evidence that DOCK180 exchange activity on Rac does not require the presence of ELMO.

21. Turner CE: Paxillin interactions. J Cell Sci 2000, 113:4139-4140.

22. Del Pozo MA, Kiosses WB, Alderson NB, Meller N, Hahn KM,

•- Schwartz MA: Integrins regulate GTP-Rac localized effector 
interactions through dissociation of RhoGDI. Nat Cell Biol 2002, 4:232-239.

Del Pozo and colleagues present a novel mechanism for the spatial control of Rac signaling in response to integrin adhesion. They provide evidence that integrin engagement initiates translocation of cytoplasmic GTP-bound Rac to the plasma membrane by RhoGDI. At the membrane, RacGTP is released from RhoGDI and is free to interact with effectors.

23. Ren XD, Kiosses WB, Schwartz MA: Regulation of the small GTP-binding protein Rho by cell adhesion and the cytoskeleton. EMBO J 1999, 18:578-585.

24. Arthur WT, Burridge K: RhoA inactivation by p190RhoGAP regulates cell spreading and migration by promoting membrane protrusion and polarity. Mol Biol Cell 2001, 12:2711-2720.

25. Arthur WT, Petch LA, Burridge K: Integrin engagement suppresses RhoA activity via a c-Src-dependent mechanism. Curr Biol 2000, 10:719-722.

26. Ren XD, Kiosses WB, Sieg DJ, Otey CA, Schlaepfer DD, Schwartz MA: Focal adhesion kinase suppresses Rho activity to promote focal adhesion turnover. J Cell Sci 2000, 113:3673-3678.

27. Tsubouchi A, Sakakura J, Yagi R, Mazaki Y, Schaefer E, Yano H,

- Sabe H: Localized suppression of RhoA activity by Tyr31/118phosphorylated paxillin in cell adhesion and migration. J Cell Biol 2002, 159:673-683.

The experiments in this paper suggest that paxillin is involved in the transient decrease in Rho activity following integrin engagement. Evidence is provided that integrin-mediated tyrosine phosphorylation of paxillin generates a binding site for p120RasGAP, thereby liberating it from association with p190RhoGAP. p190RhoGAP freed from p120RasGAP is activated and contributes to the decrease in RhoA activity.

28. Woods A, Couchman JR: Protein kinase $\mathbf{C}$ involvement in focal adhesion formation. J Cell Sci 1992, 101:277-290.

29. Saoncella S, Echtermeyer F, Denhez F, Nowlen JK, Mosher DF, Robinson SD, Hynes RO, Goetinck PF: Syndecan-4 signals cooperatively with integrins in a Rho-dependent manner in the assembly of focal adhesions and actin stress fibers. Proc Natl Acad Sci USA 1999, 96:2805-2810.

30. Bloom L, Ingham KC, Hynes RO: Fibronectin regulates assembly of actin filaments and focal contacts in cultured cells via the heparin-binding site in repeat III13. Mol Biol Cell 1999, 10:1521-1536.

31. O'Connor KL, Nguyen BK, Mercurio AM: RhoA function in lamellae formation and migration is regulated by the $\alpha 6 \beta 4$ integrin and cAMP metabolism. J Cell Biol 2000, 148:253-258.

32. Leyton L, Schneider P, Labra CV, Ruegg C, Hetz CA, Quest AF, Bron $C$ : Thy -1 binds to integrin $\beta 3$ on astrocytes and triggers formation of focal contact sites. Curr Biol 2001, 11:1028-1038.

33. Miao H, Li S, Hu YL, Yuan S, Zhao Y, Chen BP, Puzon-McLaughlin - W, Tarui T, Shyy JY, Takada $Y$ et al.: Differential regulation of Rho GTPases by $\beta 1$ and $\beta 3$ integrins: the role of an extracellular domain of integrin in intracellular signaling. J Cell Sci 2002, 115:2199-2206.

The ability of $\beta 1$ and $\beta 3$ integrins to increase Rho activity is assessed. Over-expression of $\beta 3$, but not $\beta 1$ in $\mathrm{CHO}$ cells, results in increased RhoGTP levels. Substitution of the $\beta 1$ extracellular I-domain-like sequence with the corresponding sequences from the $\beta 3$ subunit confers the ability of $\beta 1$ to activate Rho, indicating that the integrin extracellular domain is important.

34. Danen EH, Sonneveld P, Brakebusch C, Fassler R, Sonnenberg A:

-. The fibronectin-binding integrins $\alpha 5 \beta 1$ and $\alpha \mathrm{v} \beta 3$ differentially modulate RhoA-GTP loading, organization of cell-matrix adhesions, and fibronectin fibrillogenesis. J Cell Biol 2002, 159:1071-1086.

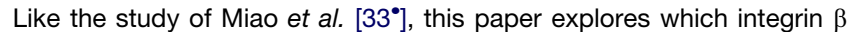
subunit is responsible for the increase in Rho activity that occurs after the initial dip following integrin ligation. Using $\beta 1$ null cells in which $\beta 1$ or $\beta 3$ are expressed, the authors find that $\beta 1$ integrins stimulate Rho activity but $\beta 3$ has no effect. This result is the opposite from that obtained by Miao et al. and may reflect the different cells lines used. In both studies, the extracellular domain determined the response.

35. Maher PA: Inhibition of the tyrosine kinase activity of the fibroblast growth factor receptor by the methyltransferase inhibitor 5' -methylthioadenosine. J Biol Chem 1993, 268:4244-4249.

36. Sastry SK, Lyons PD, Schaller MD, Burridge K: PTP-PEST - $\quad$ controls motility through regulation of Rac1. J Cell Sci 2002, 115:4305-4316.

The authors investigate the mechanism by which PTP-PEST overexpression leads to an inhibition of cell migration. They show that PTP-PEST overexpression results in decreased Rac-GTP levels and is accompanied by an inhibition of protrusive activity.

37. Garton AJ, Flint AJ, Tonks NK: Identification of p130(cas) as a substrate for the cytosolic protein tyrosine phosphatase PTPPEST. Mol Cell Biol 1996, 16:6408-6418.

38. Shen Y, Schneider G, Cloutier JF, Veillette A, Schaller MD: Direct association of protein tyrosine phosphatase PTP-PEST with paxillin. J Biol Chem 1998, 273:6474-6481.

39. Garton AJ, Tonks NK: Regulation of fibroblast motility by the protein tyrosine phosphatase PTP-PEST. J Biol Chem 1999, 274:3811-3818

40. Angers-Loustau A, Cote JF, Charest A, Dowbenko D, Spencer S, Lasky LA, Tremblay ML: Protein tyrosine phosphatase-PEST regulates focal adhesion disassembly, migration, and cytokinesis in fibroblasts. J Cell Biol 1999, 144:1019-1031.

41. Yu DH, Qu CK, Henegariu O, Lu X, Feng GS: Protein tyrosine phosphatase Shp-2 regulates cell spreading, migration, and focal adhesion. J Biol Chem 1998, 273:21125-21131.

42. Saxton TM, Henkemeyer M, Gasca S, Shen R, Rossi DJ, Shalaby F, Feng GS, Pawson T: Abnormal mesoderm patterning in mouse embryos mutant for the SH2 tyrosine phosphatase Shp-2. EMBO J 1997, 16:2352-2364.

43. Oh ES, Gu H, Saxton TM, Timms JF, Hausdorff S, Frevert EU, Kahn BB, Pawson T, Neel BG, Thomas SM: Regulation of early events in integrin signaling by protein tyrosine phosphatase SHP-2. Mol Cell Biol 1999, 19:3205-3215.

44. Manes S, Mira E, Gomez-Mouton C, Zhao ZJ, Lacalle RA, Martinez AC: Concerted activity of tyrosine phosphatase SHP-2 and focal adhesion kinase in regulation of cell motility. Mol Cell Biol 1999, 19:3125-3135.

45. Inagaki K, Yamao T, Noguchi T, Matozaki T, Fukunaga $\mathrm{K}$, Takada T, Hosooka T, Akira S, Kasuga M: SHPS-1 regulates integrin-mediated cytoskeletal reorganization and cell motility. EMBO J 2000, 19:6721-6731.

46. O'Reilly AM, Pluskey S, Shoelson SE, Neel BG: Activated mutants of SHP-2 preferentially induce elongation of Xenopus animal caps. Mol Cell Biol 2000, 20:299-311.

47. Kodama A, Matozaki T, Fukuhara A, Kikyo M, Ichihashi M, Takai Y: Involvement of an SHP-2-Rho small G protein pathway in hepatocyte growth factor/scatter factor-induced cell scattering. Mol Biol Cell 2000, 11:2565-2575.

48. Schoenwaelder SM, Petch LA, Williamson D, Shen R, Feng GS, Burridge K: The protein tyrosine phosphatase Shp-2 regulates RhoA activity. Curr Biol 2000, 10:1523-1526.

49. Sordella R, Jiang W, Chen GC, Curto M, Settleman J: Modulation -. of Rho GTPase signaling regulates a switch between adipogenesis and myogenesis. Cell 2003, 113:147-158.

This groundbreaking report shows that Rho is a key regulator of IGF-1 signals that direct the adipogenesis-myogenesis cell-fate decision. Cells derived from embryos lacking p190-B RhoGAP exhibit excessive Rho activity and are defective for adipogenesis. The level of active Rho in these cells is regulated by tyrosine phosphorylation of p190-B RhoGAP and its subcellular localization. Tyrosine phosphorylated $\mathrm{p} 190-\mathrm{B}$ is shown to be a substrate for SHP-2. Attenuation of the levels of active Rho is sufficient to alter the differentiation program of adipocyte and myocyte precursors.

50. Chikumi H, Fukuhara S, Gutkind JS: Regulation of G-proteinlinked guanine nucleotide exchange factors for Rho, PDZRhoGEF, and LARG by tyrosine phosphorylation: evidence of a role for focal adhesion kinase. J Biol Chem 2002, 277:12463-12473.

51. Suzuki N, Nakamura S, Mano H, Kozasa T: Ga12 activates Rho GTPase through tyrosine-phosphorylated leukemiaassociated RhoGEF. Proc Natl Acad Sci USA 2003, 100:733-738. 
52. Crespo P, Schuebel KE, Ostrom AA, Gutkind JS, Bustelo XR: Phosphotyrosine-dependent activation of Rac-1 GDP/GTP exchange by the vav proto-oncogene product. Nature 1997, 385:169-172.

53. Tsuda M, Matozaki T, Fukunaga K, Fujioka Y, Imamoto A, Noguchi T, Takada T, Yamao T, Takeda H, Ochi F et al.: Integrinmediated tyrosine phosphorylation of SHPS-1 and its association with SHP-2. Roles of Fak and Src family kinases. $J$ Biol Chem 1998, 273:13223-13229.

54. Hogg N, Henderson R, Leitinger B, McDowall A, Porter J, Stanley $P$ : Mechanisms contributing to the activity of integrins on leukocytes. Immunol Rev 2002, 186:164-171.

55. Lacalle RA, Mira E, Gomez-Mouton C, Jimenez-Baranda S, -. Martinez AC, Manes S: Specific SHP-2 partitioning in raft domains triggers integrin-mediated signaling via Rho activation. J Cell Biol 2002, 157:277-289.

The effect of partitioning of SHP-2 to lipid rafts is examined. The authors present evidence that targeting of SHP-2 to lipid rafts increases the basal and fibronectin-stimulated levels of Rho-GTP levels. However, ablating the phosphatase activity of the SHP-2 partitioned to lipid rafts prevents the transient inhibition of Rho.

56. Su J, Muranjan M, Sap J: Receptor protein tyrosine phosphatase $\alpha$ activates Src-family kinases and controls integrin-mediated responses in fibroblasts. Curr Biol 1999, 9:505-511.

57. Lammers $\mathrm{R}$, Lerch MM, Ullrich A: The carboxyl-terminal tyrosine residue of protein-tyrosine phosphatase $\alpha$ mediates association with focal adhesion plaques. J Biol Chem 2000, 275:3391-3396.

58. Ponniah S, Wang DZ, Lim KL, Pallen CJ: Targeted disruption of the tyrosine phosphatase PTP $\alpha$ leads to constitutive downregulation of the kinases Src and Fyn. Curr Biol 1999, 9:535-538.

59. Zeng L, Si X, Yu WP, Le HT, Ng KP, Teng RM, Ryan K, Wang DZ,

- $\quad$ Ponniah S, Pallen CJ: PTP $\alpha$ regulates integrin-stimulated FAK autophosphorylation and cytoskeletal rearrangement in cell spreading and migration. J Cell Biol 2003, 160:137-146.

Using mouse embryo fibroblasts isolated from mice null for $\operatorname{PTP} \alpha$, the role of PTP $\alpha$ is investigated in signaling pathways downstream from integrin ligation. The authors find severely decreased tyrosine phosphorylation of $\mathrm{FAK}$ and other proteins, which is consistent with PTP $\alpha$ lying between integrins and FAK in the signaling pathway.

60. von Wichert G, Jiang G, Kostic A, De Vos K, Sap J, Sheetz MP: - RPTP- $\alpha$ acts as a transducer of mechanical force on $\alpha \mathrm{v} / \beta 3$ integrin-cytoskeleton linkages. J Cell Biol 2003, 161:143-153. PTP $\alpha$ binds to integrin $\alpha v \beta 3$, but not $\alpha 5 \beta 1$, during cell spreading on fibronectin. PTP $\alpha$ is required for the force-dependent reinforcement of $\alpha \vee \beta 3$ integrin adhesions. Downstream from $\alpha \vee \beta 3$ integrin ligation, PTP $\alpha$ activates the Src family kinase, Fyn.

61. Felsenfeld DP, Schwartzberg PL, Venegas A, Tse R, Sheetz MP: Selective regulation of integrin-cytoskeleton interactions by the tyrosine kinase Src. Nat Cell Biol 1999, 1:200-206.

62. Manganello JM, Huang JS, Kozasa T, Voyno-Yasenetskaya TA, Le Breton GC: Protein kinase A mediated phosphorylation of the $\mathrm{G} \alpha 13$ switch I region alters the $\mathrm{G} \alpha \beta \gamma 13-\mathrm{G}$ protein-coupled receptor complex and inhibits Rho activation. $J$ Biol Chem 2003, 278:124-130.

63. Lang P, Gesbert F, Delespine-Carmagnat M, Stancou R, Pouchelet M, Bertoglio J: Protein kinase A phosphorylation of RhoA mediates the morphological and functional effects of cyclic AMP in cytotoxic lymphocytes. EMBO J 1996, 15:510-519.

64. Ellerbroek SM, Wennerberg K, Burridge K: Serine phosphorylation negatively regulates RhoA in vivo. J Biol Chem 2003, 278:19023-19031.

65. Lamb NJ, Fernandez A, Conti MA, Adelstein R, Glass DB, Welch WJ, Feramisco JR: Regulation of actin microfilament integrity in living nonmuscle cells by the cAMP-dependent protein kinase and the myosin light-chain kinase. J Cell Biol 1988, 106:1955-1971.

66. Howe AK, Hogan BP, Juliano RL: Regulation of vasodilatorstimulated phosphoprotein phosphorylation and interaction with $\mathrm{Abl}$ by protein kinase $\mathrm{A}$ and cell adhesion. $\mathrm{J}$ Biol Chem 2002, 277:38121-38126.

67. Howe AK, Juliano RL: Regulation of anchorage-dependent signal transduction by protein kinase $A$ and p21-activated kinase. Nat Cell Biol 2000, 2:593-600.

68. O'Connor KL, Mercurio AM: Protein kinase A regulates Rac and is required for the growth-factor-stimulated migration of carcinoma cells. J Biol Chem 2001, 276:47895-47900.

69. Feoktistov I, Goldstein AE, Biaggioni I: Cyclic AMP and protein kinase A stimulate Cdc42: role of $A(2)$ adenosine receptors in human mast cells. Mol Pharmacol 2000, 58:903-910.

70. Laudanna C, Campbell JJ, Butcher EC: Elevation of intracellular cAMP inhibits RhoA activation and integrin-dependent leukocyte adhesion induced by chemoattractants. J Biol Chem 1997, 272:24141-24144.

71. Kim S, Harris M, Varner JA: Regulation of integrin avß3mediated endothelial cell migration and angiogenesis by integrin $\alpha 5 \beta 1$ and protein kinase A. J Biol Chem 2000, 275:33920-33928.

72. Whelan MC, Senger DR: Collagen I initiates endothelial cell morphogenesis by inducing actin polymerization through suppression of cyclic AMP and protein kinase A. J Biol Chem 2003, 278:327-334.

73. He Y, Grinnell F: Stress relaxation of fibroblasts activates a cyclic AMP signaling pathway. J Cell Biol 1994, 126:457-464.

74. del Pozo MA, Price LS, Alderson NB, Ren XD, Schwartz MA: Adhesion to the extracellular matrix regulates the coupling of the small GTPase Rac to its effector PAK. EMBO J 2000, 19:2008-2014.

75. Whittard JD, Akiyama SK: Positive regulation of cell-cell and cell-substrate adhesion by protein kinase A. J Cell Sci 2001, 114:3265-3272.

76. Meyer CJ, Alenghat FJ, Rim P, Fong JH, Fabry B, Ingber DE: Mechanical control of cyclic AMP signalling and gene transcription through integrins. Nat Cell Biol 2000, 2:666-668.

77. Jaken S, Leach K, Klauck T: Association of type-3 protein kinase C with focal contacts in rat embryo fibroblasts. J Cell Biol 1989, 109:697-704.

78. Vuori K, Ruoslahti E: Activation of protein kinase $\mathbf{C}$ precedes a5 $\beta 1$ integrin-mediated cell spreading on fibronectin. $J$ Biol Chem 1993, 268:21459-21462.

79. Schlaepfer DD, Hanks SK, Hunter T, van der Geer P: Integrinmediated signal transduction linked to Ras pathway by GRB2 binding to focal adhesion kinase. Nature 1994, 372:786-791.

80. Disatnik MH, Rando TA: Integrin-mediated muscle-cell spreading. The role of protein kinase $c$ in outside-in and inside-out signaling and evidence of integrin cross-talk. J Biol Chem 1999, 274:32486-32492.

81. Disatnik MH, Boutet SC, Lee CH, Mochly-Rosen D, Rando TA:

- Sequential activation of individual PKC isozymes in integrinmediated muscle cell spreading: a role for MARCKS in an integrin signaling pathway. J Cell Sci 2002, 115:2151-2163.

The roles of individual PKC isozymes in cell spreading and FAK phosphorylation were examined. The authors demonstrate a sequential activation of PKC $\varepsilon, P K C \alpha$ and PKC $\delta$ as muscle cells adhere and spread on the ECM.

82. Schliwa M, Nakamura T, Porter KR, Euteneuer U: A tumor promoter induces rapid and coordinated reorganization of actin and vinculin in cultured cells. J Cell Biol 1984, 99:1045-1059.

83. Kellie S, Holme TC, Bissell MJ: Interaction of tumour promoters with epithelial cells in culture. An immunofluorescence study. Exp Cell Res 1985, 160:259-274.

84. Woods A, Couchman JR: Syndecan-4 and focal adhesion function. Curr Opin Cell Biol 2001, 13:578-583.

85. Mostafavi-Pour Z, Askari JA, Parkinson SJ, Parker PJ, Ng TT,

-. Humphries MJ: Integrin-specific signaling pathways controlling focal adhesion formation and cell migration. $J$ Cell Biol 2003, 161:155-167. 
This paper demonstrates that integrins differ in their requirement for PKC activation in order for them to support focal adhesion assembly. The integrin $\alpha 5 \beta 1$ requires $\mathrm{PKC} \alpha$ for focal adhesion formation, whereas $\alpha 4 \beta 1$ does not.

86. Liu S, Kiosses WB, Rose DM, Slepak M, Salgia R, Griffin JD, Turner $\mathrm{CE}$, Schwartz MA, Ginsberg MH: A fragment of paxillin binds the $\alpha \mathbf{4}$ integrin cytoplasmic domain (tail) and selectively inhibits $\alpha 4-$ mediated cell migration. J Biol Chem 2002, 277:20887-20894.

87. Longley RL, Woods A, Fleetwood A, Cowling GJ, Gallagher JT, Couchman JR: Control of morphology, cytoskeleton and migration by syndecan-4. J Cell Sci 1999, 112:3421-3431.

88. Holinstat M, Mehta D, Kozasa T, Minshall RD, Malik AB: PKC $\alpha-$ induced p115RhoGEF phosphorylation signals endothelial cytoskeletal rearrangement. J Biol Chem 2003, in press.

89. Gilmore AP, Burridge K: Regulation of vinculin binding to talin and actin by phosphatidyl-inositol-4-5-bisphosphate. Nature 1996, 381:531-535.

90. Johnson RP, Craig SW: An intramolecular association between the head and tail domains of vinculin modulates talin binding. J Biol Chem 1994, 269:12611-12619.

91. Steimle PA, Hoffert JD, Adey NB, Craig SW: Polyphosphoinositides inhibit the interaction of vinculin with actin filaments. J Biol Chem 1999, 274:18414-18420.

92. Martel V, Racaud-Sultan C, Dupe S, Marie C, Paulhe F, Galmiche A, Block MR, Albiges-Rizo C: Conformation, localization, and integrin binding of talin depend on its interaction with phosphoinositides. J Biol Chem 2001, 276:21217-21227.

93. McNamee HP, Ingber DE, Schwartz MA: Adhesion to fibronectin stimulates inositol lipid synthesis and enhances PDGFinduced inositol lipid breakdown. J Cell Biol 1993, 121:673-678.

94. Ling K, Doughman RL, Firestone AJ, Bunce MW, Anderson RA:

-• Type $1 \gamma$ phosphatidylinositol phosphate kinase targets and regulates focal adhesions. Nature 2002, 420:89-93.

This paper and that of Di Paola et al. [95.*] demonstrate that a splice isoform of PIP 5-kinase $\gamma$ is targeted to focal adhesions via an interaction with talin. As a result PIP 5-kinase is activated via phosphorylation by FAK. This provides a mechanism for the spatial generation of $\operatorname{Ptdlns}(4,5) P_{2}$ at sites of integrin engagement.

95. Di Paolo G, Pellegrini L, Letinic K, Cestra G, Zoncu R, Voronov S, -. $\quad$ Chang S, Guo J, Wenk MR, De Camilli P: Recruitment and regulation of phosphatidylinositol phosphate kinase type $1 \gamma$ by the FERM domain of talin. Nature 2002, 420:85-89.
This study is one of two that simultaneously report that talin binds to PIP 5-kinase $\gamma$ and regulates the production of Ptdlns $(4,5) P_{2}$ at sites of integrin clustering. Evidence is provided that the interaction between PIP 5-kinase and talin is mediated by the $\mathrm{N}$-terminal FERM domain of talin, and that overexpression of PIP 5-kinase, or expression of its Cterminal domain disrupts focal adhesions.

96. Schafer DA, Jennings PB, Cooper JA: Dynamics of capping protein and actin assembly in vitro: uncapping barbed ends by polyphosphoinositides. J Cell Biol 1996, 135:169-179.

97. DeMali KA, Barlow CA, Burridge K: Recruitment of the Arp2/3 - complex to vinculin: coupling membrane protrusion to matrix adhesion. J Cell Biol 2002, 159:881-891.

The authors report that membrane protrusion and cell adhesion are coupled via a direct, but transient, association of the Arp2/3 complex with vinculin. The interaction is regulated by $\mathrm{PI} 3 \mathrm{~K}$ and Rac activity and is sufficient to recruit the Arp2/3 complex to new sites of integrin adhesion and is required for efficient membrane protrusion.

98. Sturge J, Hamelin J, Jones GE: N-WASP activation by a B1-integrin-dependent mechanism supports PI3Kindependent chemotaxis stimulated by urokinase-type plasminogen activator. J Cell Sci 2002, 115:699-711.

99. Higgs HN, Pollard TD: Regulation of actin filament network formation through ARP2/3 complex: activation by a diverse array of proteins. Annu Rev Biochem 2001, 70:649-676.

100. Rohatgi R, Nollau P, Ho HY, Kirschner MW, Mayer BJ: Nck and phosphatidylinositol 4,5-bisphosphate synergistically activate actin polymerization through the N-WASP-Arp2/3 pathway. $J$ Biol Chem 2001, 276:26448-26452.

101. Eden S, Rohatgi R, Podtelejnikov AV, Mann M, Kirschner MW:

-. Mechanism of regulation of WAVE1-induced actin nucleation by Rac1 and Nck. Nature 2002, 418:790-793.

This exciting study describes a mechanism for WAVE-dependent activation of the Arp2/3 complex. In this model, WAVE1 exists in a heterotetrameric complex that includes human PIR121, NCK-associated protein with an molecular weight of 125000 (NAP125) and HSPC300. Binding of Rac-GTP or Nck causes dissociation of the WAVE1 complex, which releases active WAVE1-HSPC300 to activate the Arp2/3 complex to nucleate actin polymerization.

102. Anton IM, Lu W, Mayer BJ, Ramesh N, Geha RS: The WiskottAldrich syndrome protein-interacting protein (WIP) binds to the adaptor protein Nck. J Biol Chem 1998, 273:20992-20995.

103. Hannigan GE, Leung-Hagesteijn C, Fitz-Gibbon L, Coppolino MG, Radeva G, Filmus J, Bell JC, Dedhar S: Regulation of cell adhesion and anchorage-dependent growth by a new B1-integrin-linked protein kinase. Nature 1996, 379:91-96. 